\title{
Remote Sensing Image Fusion Method Based on improved Radial Basis and Fast partition Interpolation
}

\author{
Zhanwei Qu \\ School of Information and Electronic Engineering, Zhejiang Gong Shang University, Hangzhou \\ 310018, China \\ quzw0824@163.com
}

\begin{abstract}
A novel algorithm for image fusion based on improved Radial Basis Function named Compactly Supported Radial Basis Function (CSRBF) and fast partition interpolation is proposed. Empirical mode decomposition (EMD) construct the implicit surface for approximating the 3D points set by using RBF for reconstructing smooth surface. Using CSRBF, the matrix of corresponding system of the linear algebraic equations is spare and bounded. So it can decrease the complexity of $\mathrm{RBF}$ algorithm. A fast block method enables to improve processing speed. In addition, the paper combines IHS and à trous wavelet transform (AWT) so that spectral information of the original image is reserved and spatial detail of image fusion is enhanced. A number of examples demonstrates that the algorithm is applied to the remote sensing image fusion and obtain good results.
\end{abstract}

Keywords: Compactly supported; Empirical mode decomposition; Wavelet transform; Remote sensing image fusion.

\section{Introduction}

A single image sensor is often not able to extract enough information from the scene, so the information of multiple images needs to be fused to improve the quality of the images. Currently, more common image fusion algorithm are mainly: IHS transform, wavelet transform, principal component analysis (PCA) method, but the presence of these two algorithms disadvantages: (1). losing some useful information in the process of image fusion; (2). computing speed slow and taking up more system resources.

The joint space-spatial frequency representations have received special attention in the fields of image processing, vision and pattern recognition. Huang [1] introduces a multiresolution decomposition technique: the empirical mode decomposition (EMD), which is adaptive and appears to be suitable for non-linear, non-stationary data analysis. A new analysis method of texture images based on bidimensional empirical mode decomposition (BEMD) [2]. Compared to traditional and integral transform methods, EMD can offer better time-frequency of decomposition for non-linear, non-stationary signal, its current research focused on solving regional extreme point, solving envelope by the plane interpolation, boundary processing, screening termination conditions, fast algorithms, etc [3]. A bidimensional empirical mode decomposition method is proposed [4], which is based on the principles of morphology and radial basis function (RBF) interpolation. It is possible to reconstruct a relatively smooth surface, but the RBF will along with a large amount of computation and slow calculation speed. Delaunay triangulation interpolation is used for BEMD, which greatly reduce the amount of calculation. But its error is too large for the extreme point sparse or low-frequency extreme point fitting interpolation, so it is only suitable for high-frequency images. A method of combining IHS transform, à trous wavelet transform and EMD for remote sensing image fusion preserves the spectral information of multispectral image and enhanced spatial detail of fusion image [6]. But the EMD based on Bicubic interpolation function increase sharply for computation. A novel algorithm for image inpainting based on CSRBF interpolation is proposed to construct the implicit surface for approximating the points set interpolating the points in absent portion [7]. Using CSRBF, the matrix of corresponding system of the linear algebraic equations is spare and bounded. So it can decrease the complexity of RBF algorithm.

Based on the above, this paper presents compactly supported radial basis functions and fast block interpolation. Consider that RBF interpolation can reconstruct a relatively smooth surface but have 
many deficiencies of the large amount of calculation resulting in interpolation slower, remote sensing image fusion of the paper be treated with EMD combined with IHS transform and AWT in order to avoid solving large linear algebraic equations.

\section{Scattered Data Interpolation}

\subsection{RBF Interpolation}

For a large number of scattered points decomposed by EMD, RBF interpolation technology is currently the most widely used surface reconstruction method. Since the degree of the surface wrinkles reflects the size of the surface energy, the purpose of RBF interpolation is to get a smooth interpolation surface, and surface energy that is as small as possible.

The most important job used RBF interpolation is to gain the implicit function of interpolation, which corresponds to the mapping of the gray image point, the implicit function satisfies:

$$
s\left(t_{i}\right)=f_{i}, i=1,2, \cdots, N
$$

Where $t_{i}$ is extreme point, $f_{i}$ is gray image value corresponding to the extreme point.

Then a variational method is used as far as possible little constraint conditions in surface energy to solving formula (1) to get the general solution:

$$
s(t)=p(t)+\sum_{i=1}^{N} \lambda_{i} \phi\left(\left\|t-t_{i}\right\|\right)
$$

Where the $\lambda_{i}$ s are the RBF coefficients, $\|\bullet\|$ denotes the Euclidian norm, $\phi(\bullet)$ is the radial basis function. $p(t)$ is low degree polynomial, $p=c_{1} x+c_{2} y+c_{3}$.

As it relates to the energy functional, therefore $f \in B L^{(2)}\left(R^{2}\right)$ (Beppo-Levi space), but arbitrary choice equation (2) might cause $f \notin B L^{(2)}\left(R^{2}\right)$, so the orthogonal condition shall be added, as follows:

$$
\sum_{i=0}^{N} \lambda_{i}=\sum_{i=0}^{N} \lambda_{i} x_{i}=\sum_{i=0}^{N} \lambda_{i} y_{i}=0
$$

Combining equation (1), (2), (3), N+3 dimensional linear equations are obtained. We will gain the RBF coefficients $\lambda_{i}$ and polynomial coefficients $c_{i}$ if the known extreme points and the gray image values at the extreme points are put into the system of linear equations to be calculated. Finally, we will confirm an expression of the implicit function $s(t)$.

Substituting coordinates of all pairs of points of the two-dimensional image into the linear equation, one could obtained gray values of each coordinate point.

\subsection{Fast Block Interpolation}

RBF interpolation slow mainly due to the need to solve high-dimensional matrix, resulting in increasing the amount of calculation. If the image has $\mathrm{N}$ extreme points, we need to solve $N+3$ dimensional linear equations in a screening extremum curved surface fitting. Using a Gaussian elimination method to solve $n$ dimensional linear equations, it requires $n\left(n^{2}+2 n-1\right)$ times multiplication and division operation and $\frac{1}{2} n(n-1)(n+3)$ times addition and subtraction operation. With regard to an image, the correlation between pixels near is big, however the correlation between pixels far is small or even negligible. Therefore, the idea of RBF interpolation for the sub-regional extreme points, which is dividing into several sub-image blocks that are respectively decomposed with EMD. Because of relatively few extreme points of each sub-block, which would avoid the difficulties of solving large linear equations and handling large matrices, the amount of calculation will be reduced greatly. Figure 1 shows a comparison of multiplication and division operation for directly calculating $\mathrm{n}$ dimensional linear equations or splitting into 4 groups and 16 groups. Obviously, decomposing each block of the image partitioned with EMD reduces the amount of calculation to enhance greatly the rate of the decomposition. 


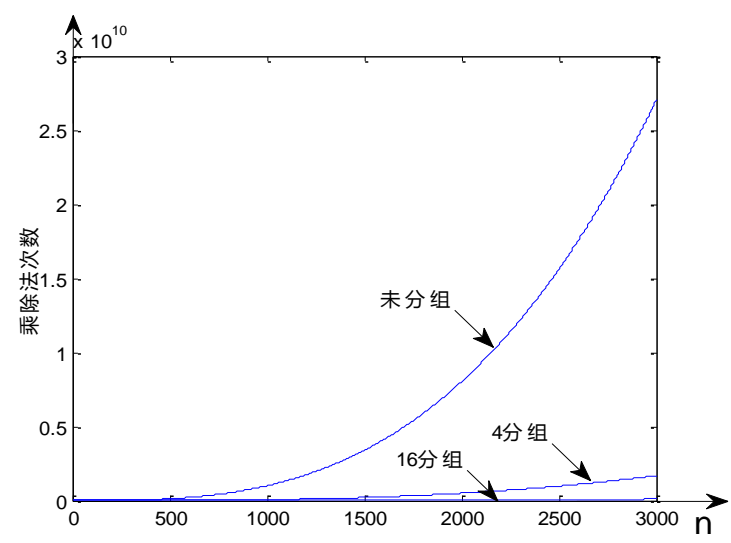

Figure 1. The Calculation of Multiplication and Division for different groups

But, a new problem will be raised by decomposing each block with EMD, which is not continuous and distortional at splicing. In order to ensure the effectiveness of the boundary data for each block of the image with RBF interpolation, each the neighboring block must have enough overlap to ensure that the combined image is not serious distortion and to suture layers IMF (IMF, intrinsic mode functions) of the adjacent block with natural suture algorithm so that the overlapping area of the adjacent block is uniform transitional part. The main idea of the algorithm is: the overlapping area of the left and right images (or the above and below images) synthesize a new image according to certain weighting. Its synthetic formula:

$$
I_{\text {new }}=I_{\text {left }} \times(1-\text { coef })+I_{\text {right }} \times \text { coef }
$$

Where $I_{\text {new }}$ is a composite image, $I_{\text {left }}$ and $I_{\text {right }}$ are respectively the left and right overlapping part, coef is weighted coefficient, $d$ is the width of the overlapping area, coef change with increments of $1 / d$ in $[0,1]$.

\section{Algorithm for Image Fusion}

In order to achieve better image fusion, one first use IHS transform [8] and AWT [9] preprocess the original image. Remote sensing image fusion process often use IHS transform to preprocess multispectral image. The image in after of IHS transform not only has high spatial resolution spectral information and retains more features to improve the accuracy of image recognition and interpretation. AWT through limited interpolation filter approximation to achieve wavelet transform without taking discrete with translation invariance. The idea is generated by the scaling function decomposition filter full-color images for details on the characteristics of the image.

Then, the application be treated by EMD image solutions, through a four-pixel neighborhood extreme point detection area of the image to be decomposed to give the corresponding extreme point image distribution. Two-dimensional image is in gray scale value highly three-dimensional surface, and the image of the extreme points is used to reconstruct the surface of discrete data points, so the space on the upper and lower envelope problem solving three-dimensional surface translate into discrete data interpolation reconstruction points.

RBF common are the global type and compactly supported type. The global type may lead non-configuration model indefinite to cause the system of equations without solution, and it does not have a compact support. To solve this problem, foreign scholars proposed CSRBF [10], who has proved to be positive definite, and its compact support so that the coefficient of linear systems have banded sparse matrix properties, which can greatly reduce the computational complexity of RBF interpolation. Remote sensing image spatial detail is more complicated and the image has more intensive extreme point, so this paper adopts CSRBF interpolation.

This paper combines with IHS transform and AWT algorithm for the multi-spectral image and panchromatic image fusion. The algorithm can get better spectrum storage capacity and higher spatial resolution. The Fusion scheme has been shown in Figure 2. 
In this paper, the specific steps image fusion algorithm is as follows:

(1) The low resolution multispectral image (LRMI) is divided into three components by IHS transform, namely intensity $(\mathrm{I})$, hue $(\mathrm{H})$ and saturation $(\mathrm{S})$, retaining the spectral characteristics of the RGB image (including hue and saturation). High-resolution panchromatic image (HRPI) is decomposed to obtain the same size and HRPI low frequency approximation surface $\left(\mathrm{H}_{\mathrm{L}}\right)$ and each high-frequency wavelet plane $\{\omega \mathrm{i}\} \mathrm{H}$ with AWT.

(2) Utilizing the algorithm of EMD, the I of the LRMI is adaptively decomposed into a series of intrinsic mode components $\{\mathrm{IMFi}\}_{\mathrm{L}}$ from high to low frequencies and the residual image component (R) through a series of processes of solving regional extreme points, settling surface interpolation, processing border distortion, screening to determine the termination condition. Similarly, on the decomposition of the low-frequency approximative surface HL for HRPI, the EMD will also give a series of $\{\mathrm{IMFi}\}_{\mathrm{H}}$.

(3) Comparison of the absolute values of a series of intrinsic mode decomposition component coefficient in step (2), whichever absolute value of the coefficient is larger the better highlight detail of $\{\mathrm{IMFi}\}_{\mathrm{F}}$ is obtained.

(4) Analysis of the R and I of LRMI and the intrinsic mode components obtained in step (3) is to gained $\{\mathrm{IMFi}\}_{\mathrm{F}}$. It performs inverse EMD (IEMD) to get a new low-frequency approximation surface instead of the low frequency component $\mathrm{H}_{\mathrm{L}}$ decomposed through AWT transformation, then conducts inverse AWT transformation (IAWT) to obtain a new image luminance component (Inew) containing high-frequency section namely spatial features of the HRPI to replace spatial feature of the LRMI that is characteristic of the I. Finally, the final fusion image is obtained with an inverse IHS transform (IIHS).

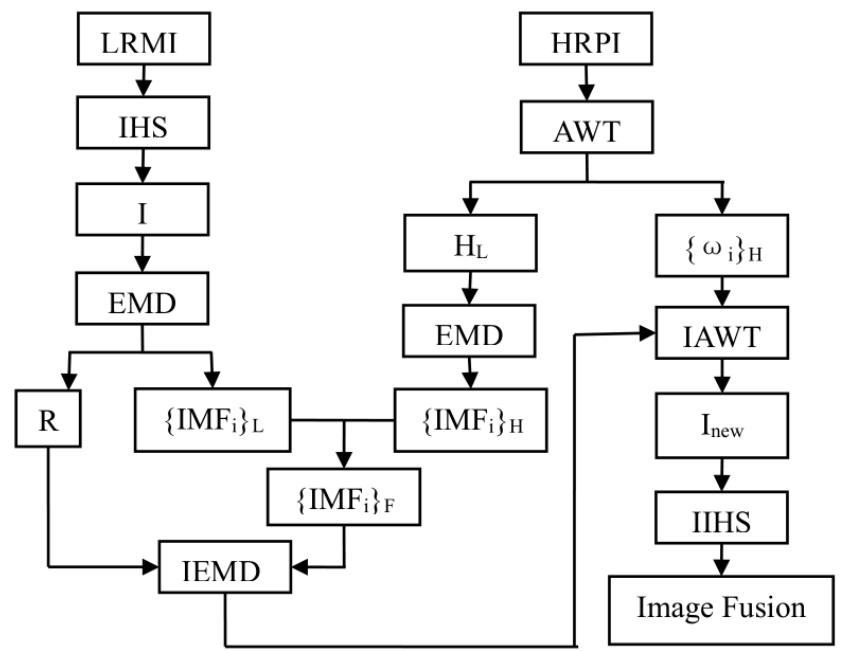

Fig. 2 the Method of the Image Fusion of the Paper

\section{Experimental Results and Analysis}

In order to verify the effectiveness of the proposed fusion method, the paper use a low-resolution multispectral image (LRMI) and a high resolution panchromatic image (HRPI), which cover Wuhan City, China, approximately from $30^{\circ} 12^{\circ}$ to $30^{\circ} 40^{\circ}$ south latitude and $113^{\circ} 35^{\circ}$ to $114^{\circ} 15^{\circ}$ west longitude. Fig. 3 displays despeckled LRMI of size $1024 \times 1024$, Fig. 4 shows true color composite. With MATLAB R2013a in experimentation, scale decomposition filter of '9-7' is used, and two IMFs are gained by the decomposition of the EMD, and the extreme points area size of 4 is selected, and the end condition of the screening process is 0.2 . 


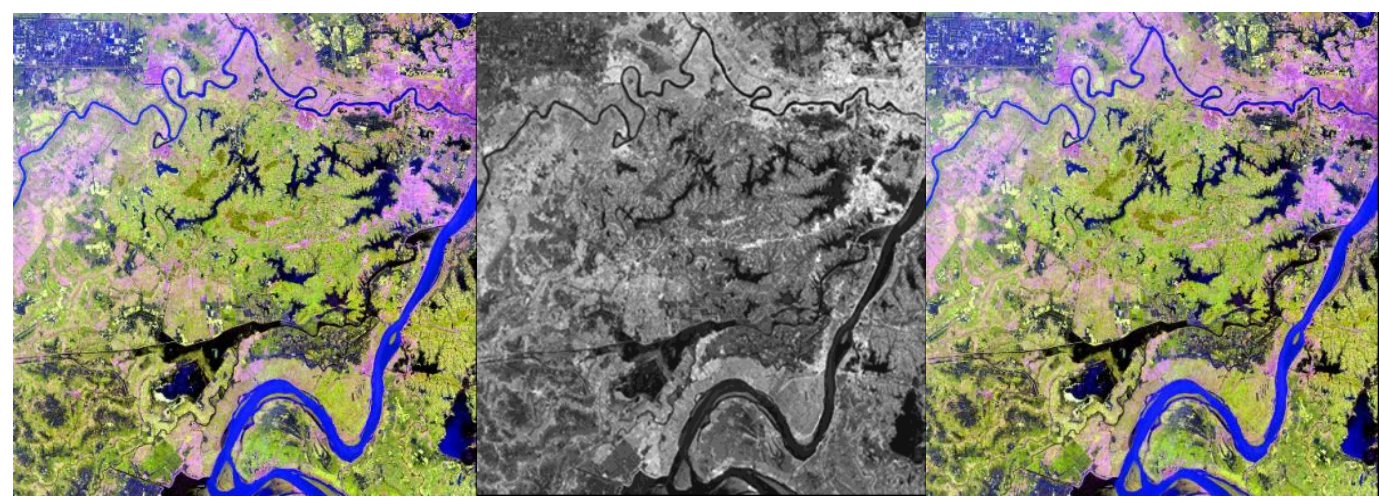

Fig. 3 LRMI

Fig. 4 HRPI

Fig. 5 AWT

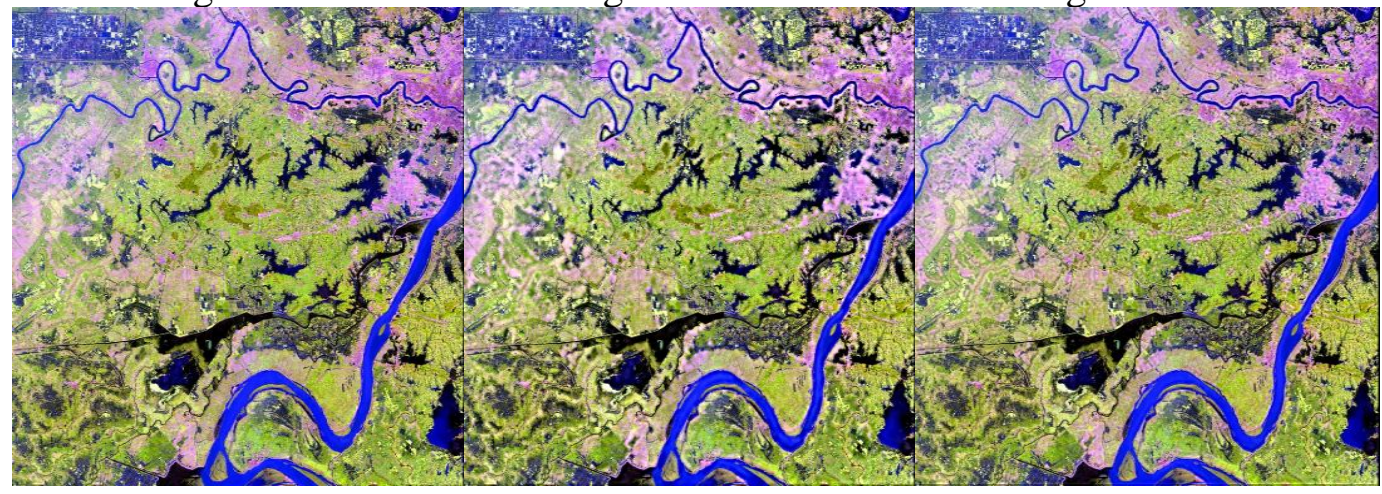

Fig. 6 AEM

Fig. 7 IAEM

Fig. 8 the paper

This paper selects three different fusion algorithms compared in this article. Fig. 5 shows AWT for image fusion [11]. Fig. 6 shows the algorithm combining AWT and EMD (AEM) for remote sensing image fusion [12]. In order to retain the spectral characteristics of LRMI, The IHS method first handle three bands. Fig. 7 displays image fusion combining AWT and EMD (IAEM) again [6]. Fig. 8 shows image fusion result with the algorithm proposed by this paper.

To further demonstrate the superiority of the proposed algorithm, we use the objective evaluation to further analyze the images. These objective evaluation: information entropy (IE) indicates how much entropy contained in the image, entropy of the greater indicates the richer integration of the image information; standard deviation (SD) reflects the discrete circumstances of the image intensity with respect to the average gray, which is used to evaluate the size of the image contrast, and the image gray level distribution is more scattered if the standard deviation is large, vice versa; average gradient $(\mathrm{AG})$ reflects the improvement in the quality of image fusion for image contrast and fine detail texture characteristics transformed, and higher-resolution images, fine details and textures will be reflected the better if average gradient is larger; correlation coefficient (CC) represents the correlation between the two images, and the greater the correlation coefficient that is closer to 1 , indicating their strong correlation; warp (WR) is used to describe the deformation of the fused image opposite of the original image distortion, and value is smaller, which reflects the smaller the spectral distortion, the smaller the degree of distortion and the better integration; mean (M) is the average of the entire gray image, which reflects the average image brightness, and the good visual effect will be reflected if the mean is moderate. Correspond to Fig. 5, Fig. 6, Fig. 7 and Fig. 8 in different fusion methods, the evaluation results are shown in Table 1.

As can be seen from Table 1, the objective indicators of image fusion algorithm basically comparative advantages, including the large amount of information of the original image, high-definition. Because the algorithm preserves the surface of each high-frequency wavelet of the high-resolution panchromatic image, it is with respect to the low-resolution multi-spectral images of the correlation coefficient is small. 
Table 1 Different Fusion Evaluation

\begin{tabular}{|c|c|c|c|c|}
\hline & AWT & AEM & IAEM & The Paper \\
\hline IE & 7.8724 & 7.8683 & 7.7934 & 7.8694 \\
\hline SD & 0.2588 & 0.2587 & 0.2844 & 0.2825 \\
\hline AG & 0.0810 & 0.0817 & 0.0844 & 0.0891 \\
\hline DD & 0.0162 & 0.0325 & 0.0392 & 0.0253 \\
\hline $\begin{array}{c}\text { CC } \\
\text { (versus LRMI) }\end{array}$ & 0.9909 & 0.9960 & 0.9665 & 0.9873 \\
\hline M & 0.5252 & 0.5251 & 0.5241 & 0.5243 \\
\hline
\end{tabular}

\section{Conclusion}

This article will use a block-based interpolation and Compactly Supported Radial Basis Function method for image fusion, through a series of non-uniform discrete sampling points reconstructing smooth surfaces, not only reconstructing the missing information, but also reducing the surface interpolation algorithm complexity and computational capacity. Experiments show that, image fusion based on CSRBF and fast partition interpolation can get a clear picture of the integration and improve greatly operation speed.

\section{Acknowledgements}

National Natural Science Foundation (NO: 61374022).

\section{References}

[1] Huang N E, Shen Z, Long S R. The empirical mode decomposition and the Hilbert spectrum for nonlinear and non-stationary time series analysis [J]. Proc. R. Soc Lond.A, 1998, 454: 903-995.

[2] Zhou X. Bidimensional Empirical Mode Decomposition (EMD) Used in Image Processing [D]. Huazhong University of Science and Technology, 2007.

[3] Xu X G, Xu G L, Wang X L. Application about Empirical Mode Decomposition (EMD) [J]. Acta Electronica Sinica, 2009, 37 (3):581-585.

[4] Nunes J C, Guyot S, Deléchelle E. Texture analysis based on local analysis of the Bidimensional Empirical Mode Decomposition[J]. Machine Vision \& Applications, 2005, 16(3):177-188.

[5] Qin H Y. Multispectral Image Fusion Based on EMD [D]. Huazhong University of Science and Technology, 2008.

[6] Chen S, Zhang R, Su H, et al. SAR and Multispectral Image Fusion Using Generalized IHS Transform Based on à Trous Wavelet and EMD Decompositions[J]. IEEE Sensors Journal, 2010, 10(3):737-745.

[7] Wang W H, Qin X J. The Image Restoration Algorithm Based on Compactly Supported Radial Basis Function Interpolation [J]. Journal of Electronics \& Information Technology, 2006, 28(5):890-894.

[8] Choi M. A new intensity-hue-saturation fusion approach to image fusion with a tradeoff parameter [J]. Geoscience \& Remote Sensing IEEE Transactions on, 2006, 44(6):1672-1682.

[9] Ayan Seal, Debotosh Bhattacharjee, Mita Nasipuri. Human Face Recognition using Random Forest based Fusion of A-trous Wavelet Transform Coefficients from Thermal and Visible Images [J]. AEU - International Journal of Electronics and Communications, 2016. 
[10] Morse B S, Yoo T S, Chen D T, et al. Interpolating Implicit Surfaces from Scattered Surface Data Using Compactly Supported Radial Basis Functions[C]. In Proceedings of Shape Modeling Conference, Genoa, Italy, May 2001:89-98.

[11] Wang Z, Ziou D, Armenakis C, et al. A comparative analysis of image fusion methods[C]. IEEE Transactions on Geoscience and Remote Sensing. 2005:1391-1402.

[12]Chen S, Su H, Zhang R, et al. Fusing remote sensing images using à trous wavelet transform and empirical mode decomposition[J]. Pattern Recognition Letters, 2008, 29(3):330-342. 